GENERALIZATIONS OF COMPLEX ANALYSIS

BANACH CENTER PUBLICATIONS, VOLUME 37

INSTITUTE OF MATHEMATICS

POLISH ACADEMY OF SCIENCES

WARSZAWA 1996

\title{
PARTICLES, PHASES, FIELDS
}

\author{
L. WOJTCZAK, A. URBANIAK-KUCHARCZYK, \\ I. ZASADA and J. RUTKOWSKI \\ Solid State Physics Department \\ University of Eódź \\ ul. Pomorska 149/153, PL-90-236, Lódź, Poland
}

\begin{abstract}
The physical properties of particles and phasesare considered in connection with their description by means of the deformation of space-time. The analogy between particle trajectories and phase boundaries is discussed. The geometry and its curvature is related to the Clifford algebraic structure whose construction in terms of the theory of deformation leads to the expected solutions for correlation functions referring to spectroscopy and scattering problems. The stochastic nature of space-time is reflected by fluctuations of distances and time intervals.
\end{abstract}

1. Introduction. In order to construct the mechanics we need to determine the space-time when a particle and its movement is considered. The physical fields are also embedded into the space-time and their nature is usually related to the deformation of geometry. Moreover, the phases can be regarded as the fields whose parametrization corresponds to the solutions minimizing the field energy, i.e., the field variation vanishes. Thus, we can see an analogy between the particle trajectories and the phase boundaries description, which allows us to expect the interdependence of inhomogeneous systems and their geometric and algebraic structures.

First of all we consider physical aspects of the geometry confined to the four-dimensional space-time and its curvature in the context of the algebraic structure of particle trajectories. The considerations can be based on the model introduced in [6]. The spacetime is then assumed as a projection of the five-dimensional space-time with the fifth dimension being of stochastic behaviour.

From the mathematical point of view the four-dimensional space-time is described by Minkowski space $M_{4}$ which is the four-dimensional flat pseudoriemanian manifold with signature 2. The physical phenomena appearing in $M_{4}$ can be observed in the threedimensional space $R^{3}$ which is one of the projections of $M_{4}$ changing additionally in time. The model extended for five-dimensional manifold $M_{5}$ with signature 3 or 1 , in analogy

1991 Mathematics Subject Classification: Primary 70G05; Secondary 82B26, 58H15.

The paper is in final form and no version of it will be published elsewhere. 
to the relations between $M_{4}$ and $R^{3}$ allows us to understand the nature of phenomena observed in $M_{4}$ while they appear in fact in $M_{5}$. We repeat here a very illustrative example which is well known and usually reported in the theory of relativity. Namely, the contraction of a segment length in $R^{3}$ is in fact explained by the rotation of a segment in $M_{4}$ when we observe its projection in $R^{3}$ corresponding to the contracted length. A similar relation takes place between the time intervals measured by the observers situated in two coordinate systems moving in relation to each other.

The theory proposed at the geometrical level allows us to formulate the generalised wave equations whose algebra refer to the description of the eigenfunctions and energy eigenvalues in the conditions of deformed manifolds and their curvatures. In particular, the equation of a particle embedded in the five dimensional space-time can be formulated in the Kałuża-Klein or Penrose-type models whose fundamentals of local geometry for Hurwitz pairs of manifolds with bidimension $(8,5)$ characterise the physical deformation of fields.

In terms of classical mechanics the particle trajectories satisfy the variational equations which are equivalent to the equations of motion in which the deformation is taken into account in the form of effective fields determined by the connections related to the curved space-time metric.

The variational principle leads also to the Euler-Lagrange equations minimizing the thermodynamic potentials when we discuss the problem of phases coexistence in their equilibrium (cf. [3]). The thermodynamic functions are then treated as physical fields associated to the order parameters whose parametrization corresponds to the particle trajectory. On the other hand the formal analogy occurs in the case when we compare the behaviour of a system described by thermodynamic functions or elastic fields leading to the appearance of effective forces in the form of Poisson's brackets equivalent to the connection term of the covariant derivative with respect to the coordinates in the deformed, locally inhomogeneous medium.

2. Particle trajectories. The classical mechanics allows us to formulate the equations of motion by means of the Hamilton equations or the Euler Lagrange variational equation, which describe the particle trajectories as solutions minimizing the properly determined functional with respect to the most probable path of a particle. Two approaches are based on the variation of one of two functionals: the Lagrange functional $\mathcal{L}$ or the Hamilton functional $\mathcal{H}$ which are related to each other as $\mathcal{H}=\nu(\partial \mathcal{L} / \partial \nu)-\mathcal{L}$, where $\nu$ is the velocity in the space-time $(x, t)$.

In the case of deformed geometry the variational principle is defined by means of the space-time invariant $d s=-g^{i k} d x_{i} d x_{k}$ considered in a metric $g^{i k}$ applied to the description of the interaction fields in terms of the space curvature. A typical and illustrative example is then connected with the trajectories in the general theory of relativity where they are given by the geodesic lines.

Within the conventional approach to the deformation the properties of the deformed space-time can be reflected by means of covariant derivatives $d / d t \mapsto d / d t+\Gamma$ where $\Gamma$ stands for the connection of the space related to the Christoffel coefficients constructed 
on the basis of the metric elements. The Lagrange function is then given by

$$
\mathcal{L}=\frac{1}{2} m(\dot{R}+\Gamma R)^{2}-V(R)
$$

with the potential $V(R)$ which has its usual meaning and $\mathrm{m}$ standing for the mass of the considered particle. The motion of a free particle corresponds to the case $V(R)=0$ when the equation of motion reads

$$
\ddot{R}-\Gamma^{2} R=0
$$

for $\Gamma$ assumed to be independent of $R$.

Taking into account that $\nu=\dot{R}$ the Hamiltonian function is of the form

$$
\mathcal{H}=\frac{P^{2}}{2 m}-\Gamma R P
$$

with $P=m(\dot{R}+\Gamma R)$ hence the Hamilton equations

$$
\dot{R}=\frac{P}{m}-\Gamma R ; \quad \dot{P}=\Gamma P
$$

lead to Eq. (2), confirming it in a self-consistent way. The solution of Eq. (2) for $\Gamma=i \omega$ takes its form $R=R_{0} \cos \omega t$ with $R_{0}$ denoting the amplitude of oscillations.

The obtained result seems to be of great importance for the interpretation of fundamental properties of a free particle behaviour. We can see that the non-interacting particle oscillates at the vicinity of its initial position; it moves in the conditions when it should remain in rest. From the physical point of view the result can be interpreted as the action of an additional force due to the influence of the curved space-time.

The presented result can be also discussed in frames of the physical models based on the non-commutative geometry. One of them is based on the statement that probabilistic interpretation of quantum mechanics causes an unitary time evolution of the physical system due to the choice of the $q$-deformed algebra of observables with consequence on the level of dynamics. In the case of deformation described by the commutation rules $R P-q^{2} P R=i \hbar q$ the Heisenberg equations are written as [10]

$$
\dot{R}=\frac{i}{\hbar}\left(1-q^{4}\right)\left(\frac{P^{2}}{2 m}\right) R+q\left(1+q^{2}\right) \frac{P}{2 m} ; \quad \dot{P}=0
$$

hence we can see that Eq. (5) takes the form (2) for $R \rightarrow R+\bar{R}$ with the frequency

$$
\omega=\frac{1}{\hbar}\left(1-q^{4}\right)\left(\frac{P^{2}}{2 m}\right)
$$

and the shift constant $\bar{R}=\frac{i \hbar q}{\left(1-q^{2}\right) P}$ which do not vanish for $P$ being a constant with respect to (5), and $q \neq 1$ corresponding to the $q$-deformed algebra. The frequency (6) vanishes for the standard quantum mechanics $q=1$. The constant $P$ can be in fact of an arbitrary value. Thus, we can expect a spectrum of oscillations whose appearance is governed with respect to $\omega$ and $\bar{R}$. It allows us to suppose that the distribution with respect to the $P$ is of Gaussian type. Thus, the movement of a free particle embedded in the deformed space-time is of stochastic character.

3. Five dimensional construction. The proposed picture is consistent with the construction in the five dimensional space-time with a stochastic dimension [6]. The 
model leads then to the variational principle $\delta S=0$ for $S=\alpha \int d s$, in analogy to the geodetic trajectories, with the Lagrange functional $\mathcal{L}(\nu=\dot{R}, R)$ in the form

$$
L=-m c^{2}\left(1-\frac{\nu^{2}}{c^{2}}-\eta \frac{\sigma^{2}}{c^{2}}\right)^{1 / 2}
$$

where $\sigma=\sigma(R)$ represents a stochastic distribution of deformation. We assume

$$
\sigma(R)=\sum_{n} \sigma_{n} \cos \left(\omega_{n}\right) R
$$

for $\eta=1$ with the normalization condition $\sigma(0)=1$ while the origin $R=0$ is chosen arbitrarily due to the symmetry of the space. In the case of the nonrelativistic limit the variational principle reads

$$
S=\int_{0}^{\infty}\left[\frac{1}{2} m \dot{R}^{2}+\frac{1}{2} m \sigma^{2}(R)\right] d t
$$

hence the equation of motion takes its form

$$
\ddot{R}-\left(\sigma(R) \frac{d \sigma(R)}{d R} \frac{1}{R}\right) R=0
$$

hence

$$
\ddot{R}+\left(\sum_{n n^{\prime}} \sigma_{n} \sigma_{n^{\prime}} \omega_{n^{\prime}} \cos \omega_{n} R \frac{\sin \omega_{n^{\prime}} R}{R}\right) R=0
$$

reduces to

$$
\ddot{R}+\left(\sum_{n} \sigma_{n} \omega_{n}^{2}\right) R=0
$$

for $R$ sufficiently close to zero. The case $\eta=-1$ requires the distribution $\sigma(R)=$ $\sum_{n} \sin \left(\omega_{n} R\right)$ instead of (8), which leads to the initial condition $\sigma(0)=0$, but $\sum_{n} \sigma_{n}=1$. As a consequence, we obtain Eq. (12) for $\eta=-1$ which is also valid for $\eta=1$.

We can see that the solution of Eq. (2) is confirmed by means of the discussion concerning Eq. (12) derived in the geometrical way. The origin of a free particle is not a point but the space-time area where the chaotic movement is strongly localized.

4. Topology and fluctuations. Scattering and spectroscopy. The problem of deformation can be discussed at variations levels of structure. The first step was connected with the behaviour of a free particle occurring in the space-time when the deformation is introduced at the level of the space itself. The next point concerns the behaviour of a particle embedded into physical fields of interactions which can be related to the deformations influencing the considered fields.

One of the most important and characteristic situations where the deformation plays the essential role refers to the description of the surface topography. The interest in the investigations of the surface roughening phenomenon is both academic and practical. From the fundamental point of view its description allows us to relate the theoretical predictions to experiments, while the models can reflect the fundamental structure also including the subatomic deformations of the space-time. The considerations are based on topological correlations whose Fourier transforms at the surface are the standing point for calculations of the measured quantities in the experimental studies. The low energy 
electron diffraction and its different variants as diffuse or tensor low energy electron diffraction is the main technique which is used to investigate the geometrical structure of the surface [7, 12]. Almost direct mapping of the surface inhomogeneities is observed by means of the scanning tunnelling microscope which turns out a very sensitive tool for local investigations.

The backscattering electron intensities are the sum of the coherent and incoherent parts which are connected with the perfect translational symmetry of the surface layer and with the spontaneous deformation, respectively. The general expression for the incoherent intensity at the disordered surface is determined by the scattering cross section for the potential characterised by the topological correlations which reflect the fluctuations at the level of nanophase structures. In the model solid on solid the roughness of the surface contributing to the deformation of the considered space at the nanoscale level is represented by the deviations of the square average values of the (0.1) occupation operators from their mean square values. Thus, we can see that the deformation even in the nanoscale level is of statistical character. In this case the average values of the considered operators are determined by the algebra of the pseudospin operators which are connected with the occupation of the surface sites. The deformation is them given by means of several, at least, two states of configuration for the considered particles.

The investigations of the surface topology show that the correlations play an important role of the potential responsible for the incoherent processes which are connected with the deformation of the perfect crystallographic symmetry.

First of all, the remark is confirmed by the research concerning the critical scattering of neutrons by ferromagnets [4]. In order to stress that the property is of general character we discuss another example: the contribution of magnetic moments correlations to the electric resistivity in magnetic materials with the fluctuating exchange interactions. From the physical point of view the considered contribution appears in ferromagnets due to the interaction between localized spins and delocalized conduction electrons. Taking into account that the influence of magnetic bands on the conduction electrons behaviour can be described by means of the short range s-d type exchange integrals the magnetic contribution to the electrical resistivity is given by the Fourier transform of the spin correlation functions. The deformation of the magnetic correlations is introduced by the disorder of the sample which is represented by the fluctuations of the exchange parameter changing it by a random value with respect to the average value in different lattice sites [11].

The temperature and angular characteristics of the conductivity are then discussed. A comparison of the results obtained for various distributions of deformation allows us to conclude that the Gaussian function used for the description of the exchange integral fluctuations instead of a particular distribution produces similar results only for small amplitudes of fluctuations while for large fluctuations the results become different than those obtained previously. We can see that in the case of highly disordered materials the proper choice (of the distribution function) is of primary importance and it is quite possible that additional improvements of the distribution are necessary. The above conclusion is of a general character and confirms the considerations in paper [5] devoted 
to the Clifford analysis including Riemannian geometry used to the description of the electromagnetic field and its interaction with particles.

Another example showing the importance of correlations connected with the deformation of magnetic symmetry due to the randomness of spin orientations is the problem of the line-width of elementary magnetic excitations. The basic quantity in calculations of line profiles is the density of magnon states. Calculations based on the standard Green function technique lead to the approximation of the infinite life-time of magnons. The profiles of spin waves resulting from the magnon-magnon interactions, their collisions with other quasi particles and time-dependent configurations can be obtained in the frame of the coherent potential approximation. In this method the coherent potential which can be treated as an effective internal field additive to a field along the magnetization direction leads to the finite line-width of spin waves profiles via the proper choice of the two-particles Green functions which allow us to calculate the physical characteristics of the system.

One of them is related to the spectroscopy which is determined in fact by two factors: (1) the energy eigenvalues and (2) the density of states. The collection of the first factor set is usually given by the wave equation and its eigenvalues. The second factor determined by the density of states whose best fit to the experimental results is a very fruitful tool of the spectrum analysis needs the energy eigenvalues and their probabilities of appearance described by the square of the eigenfunctions. Both of the required characteristics can be achieved just by the considerations in the language of the thermodynamic Green's functions. The direct bridge between the proper Green's function and the density of states is known and important for the presented investigations.

The problem of the spectral analysis is reduced to the discussion at two levels. The atomic and nanostructural level corresponds to the discussion of the density of states introduced by the Green's functions which should be considered as a linear combination of the Gaussian-type spectral lines. The subatomic level corresponds to the confrontation of the wave equation containing the terms of stochastic nature. In the simplest case we can discuss the description of the hydrogen atom which allows us to understand the role of the space deformation.

The description at the level of the Dirac-like equation shows that the stochastic deformation removes the singularity of the Coulomb potential at its origin. This fact has its fundamental influence on the hydrogen spectrum. However, the examples of a more complex spectrum [e.g. 2] are very instructive when we consider the Gaussian analysis and we expect the stable solutions for which the linewidth reflects the deformation. In this case the spectral pictures which are complicated from their origin are good examples for the applications of the methods which consist in the separation of individual excitations.

In the context of the present considerations we would like to stress that the discussion of the role of deformation at the level of particle systems is connected with the statistic properties and their description reduces to the statistical distribution reflecting the spatial and temporal correlations between interacting particles. However, the influence of fluctuations can been how considered in the natural way in connection with the de- 
formation of stochastic character at the space-time deformation level whose appearance implies local fluctuations deforming the behaviour of statistical fluctuations.

5. Phase characteristics. The role of deformation at the macroscopic level can be exemplified by the considerations of the thermodynamic properties in the region close to the phase transitions. The thermodynamic parameters and functions discussed in the case of a sample confined by the surface can be of inhomogeneous distribution due to the symmetry breaking at the surface. The description of the order parameters is then analogous to the theory of the particle variational principle we return to the case when the fluctuations of stochastic nature concern the space. Taking into account this analogy we can write the fundamental $F$ with respect to the order parameter $\mathrm{M}$ which depends now on the distance from the surface measured along the axis $Z$, namely $[8,9]$.

$$
F[M(z)]=f_{s}\left(M_{s}\right)+\int_{0}^{\infty}\left[f(M)+\frac{1}{2} J\left(\frac{d M}{d z}\right)^{2}\right] d z
$$

where $f(M)$ denotes the potential of the bulk system described by the homogeneous order parameter given by $\delta f(M)=0$. The function $f_{s}(M)_{s}$ stands for the description of the boundary condition at the surface where $M_{s}=M(z=0)$. The coefficient $J$ plays the role of the stiffness constant responsible for the inertion of the system with respect to the applied deformation in analogy to the mass in the case of the particle movement.

From the physical point of view the phase transition theories show that the formal analogy has its deeper interpretation. The phase diagram is usually established by the minimization procedure of the thermodynamical potential $F(T ; M, H)$ with respect to the order parameter $M(T ; H)$ which is conjugated with an external field $H$ at temperature $T$. The phase $\sigma$ is then determined by the solution of the equation $\delta F(M)=0$ which can be found in the form $M_{\sigma}=M_{\sigma}(T ; H)$. In the case when the variational equation has more than one solution and the solutions are different at some intersection interval of temperature i.e. $M_{\sigma} \neq M_{\sigma^{\prime}}$ for $T \in\left(T_{\sigma}, T_{\sigma^{\prime}}\right)$ we can consider the appearance of the most probable phase and discuss the phase transition $\left(\sigma \leftrightarrow \sigma^{\prime}\right)$ determined by the physical condition: $F\left(M_{\sigma}\left(T_{c}\right)\right)=F\left(M_{\sigma^{\prime}}\left(T_{c}\right)\right)$ for the phase transition temperature $T_{c} \in\left(T_{\sigma}, T_{\sigma^{\prime}}\right)$ where $T_{\sigma}$ and $T_{\sigma^{\prime}}$ stand for the stability temperatures of the phase $\sigma$ and $\sigma^{\prime}$, respectively. The stability points satisfy the equations $\delta^{2} F\left(T_{\sigma}: M_{\sigma}\left(T_{\sigma}\right)\right)=0$, respectively. The phase transition between two phases is considered as discontinuous when the phase coexistence region exists $T_{\sigma} \leq T_{c} \leq T_{\sigma^{\prime}}$ while the transition is continuos when $T_{\sigma}=T_{c}=T_{\sigma^{\prime}}$. The existence of the surface leads to the boundary conditions at the surface for the solution corresponding to the most probable profile of the order parameter $M$ when it becomes inhomogeneous but it describes the same phase at the surface and in the volume of a sample. The profile $M=M(z)$ corresponds now to the particle trajectory $x=x(t)$.

We can also expect another situation when the surface order represents a new phase which can coexist with that inside a sample. In this case, the surface phase is described by the energy corresponding to the surface order parameter whose appearance is preferable in some interval of temperature. The analogy to the particle behaviour indicates a situation when there are two possible trajectories determined by some characteristics of the field in which the movement is considered. The particle chooses the favourable trajectory. The 
change of trajectories often manifested by the hoping between trajectories, means then the phase transition at the one particle scale.

The deformation of thermodynamic functions can be introduced on two levels discussed in connection with the space-time, i.e., one particle level, or in connection with the statistical averaging, i.e., particle ensemble level. The second case is usually accepted and its description is related to the approach of correlation functions. The first situation leads to a more original model which is closely formulated within the Clifford algebra.

The considerations of geometrical deformation of the space time allow us to replace the euclidean structure of the space or the pseudo-euclidean structure of the space-time by the curved pseudo-riemannian space whose metric generates the Christoffel symbol $\Gamma$ according to the relation

$$
\frac{d M}{d z} \mapsto \frac{d M}{d z}-\Gamma M
$$

The functional $\Gamma(M(z))$ given by (22) can be rewritten in the form [1]

$$
F[M(z)]=f_{s}\left(M_{s}\right)+\int_{0}^{\infty}\left[f(M)+\frac{1}{2} J \Gamma^{2} M^{2}+\frac{1}{2} J\left(\frac{d M}{d z}\right)^{2}\right] d z+\frac{1}{2} J \Gamma\left(M_{s}^{2}-M_{c}^{2}\right)
$$

where $M_{s}=M(z=0)$ and $M_{c}=M(z=\infty)$.

The connection $\Gamma$ can be reduced to a constant being the structural Clifford algebraic constant exactly as in the case of particle systems when investigating also surface effects introduced by the surface stochasticity. The variational conditions read

$$
\begin{gathered}
\frac{\partial f}{\partial M}+J \Gamma^{2} M=J\left(\frac{d^{2} M}{d z^{2}}\right) \\
\frac{\partial f_{s}}{\partial M}+J \Gamma M_{s}=J\left(\frac{d M}{d z}\right)_{z=0}
\end{gathered}
$$

with the boundary conditions

$$
\left(\frac{d M}{d z}\right)_{z \rightarrow \infty}=0
$$

We can see that the constant $\Gamma$ characterises the geometry introduced by fluctuating, the inhomogeneous nature of the space and it plays simultaneously an important role in the problem of stability of the systems with restricted dimensions. One of the results is of particular interest. It turns out that for $f(M)$ which does not give the stable phase $M=0$ the deformation $\Gamma$ can assure the stability of the phase $M=0$ while the phase $M=M_{c}$ is shifted to $M<M_{c}$. Moreover, the deformation creates a phase transition.

The problem which remains open concerns the evaluation of $\Gamma$. The ways based on the experimental fitting lead to different values. Therefore, it seems to us that the evaluation of $\Gamma$ should be done at the fundamental level connected with the energy and impulse fluctuations. However, the influence of fluctuations can be considered now in the natural way in connection with the deformation stochastic character at the space-time deformation level whose appearance implies the local fluctuations deforming the statistical approach.

6. Conclusions. The investigations of deformations considered in physical objects in various aspects show that the nature of deformation is connected with the structure of the 
space-time which should be extended to five dimensions where the fifth axis corresponds to the stochasticity of four-dimensional space-time projections. The conclusion of the present paper that a free particle is not localized at the geometrical point but it vibrates stochastically around this point can be interpreted as a fluctuation of the space-time which is perfectly explained in the light of the stochastic dimension projected to the four-dimensional manifold of observation. Of course, by this fact justifies the appearance of stochastic forces and inhomogeneities of dynamic character in various, real physical systems.

The model of fluctuating space-time becomes a basis for the interpretation of local fluctuations considered in thermodynamic theories where the occurrence of local fluctuations is assumed a priori at the construction of hamiltonian for a given system, independently of the other features of the hamiltonian. The local fluctuations are then distinguished from other dynamic inhomogeneities which appear in the description of a system as a result of dynamic lows governing a system assigned by the considered Hamiltonian. The present interpretation of the space-time nature seems to be confirmed by the considered here examples and formulas well as physical analogies between the described approaches in context of the role of deformation.

The present contribution brings new approaches to the application of the Clifford algebra and its geometrical consequences in physics as well as some predictions for the research in future. The main idea is that the space-time can be of stochastic nature. The applications concern then different physical phenomena whose nature seems more clear when interpreted in terms of stochasticity independently of their variety, while the examples taken from the surface science are more suitable for the sake of presentation.

The presented considerations have been based on numerous discussions which the authors held on different occasions with their colleagues: J. Lawrynowicz, O. Suzuki, C. Surry, J. Rembieliński, H. Kibler, B. Gaveau, J. Gnatenko, F. L. Castillo Alvarado, G. Contreras Puente, L. Papaloucas. We are convinced that this paper would not have been possible without their fruitful and critical comments.

The authors are grateful to the organisers of the Conference for including the topic of the present paper into the Conference schedule.

\section{References}

[1] F. L. Castillo-Alvarado, G. Contreras-Puente, J. Eawrynowicz and L. Wojtczak, A Hurwitz-Pair Approach to the Premelting Problem, in: Deformations of Mathematical Structures, J. Lawrynowicz (ed.), Kluwer Academic Publishers, Netherlands, 1994, 289-298.

[2] Yu. P. Gnatenko, J. H. Rutkowski and G. Contreras-Puente, Optical spectra of CdTe crystals doped with Co atoms, Bull. Soc. Sci. Lettres Łódź, Sér. Réch. Deform., (submitted for publication).

[3] H. Haken, Synergetics, An Introduction, Nonequilibrium Phase Transitions and Self Organization in Physics, Chemistry and Biology, Springer Verlag, Berlin, Heidelberg, New York, Tokyo, 1983. 
[4] J. Kociński and L. Wojtczak, Critical Scattering Theory. An Introduction, Phase Transition Phenomena 1, Elsevier Scientific Publ. Co.-PWN, Amsterdam, Oxford, New York, Warszawa, 1978.

[5] J. Ławrynowicz, J. Avendano-Lopez, F. L. Castillo-Alvarado, R. A. Barrio and A. Urbaniak-Kucharczyk, Clifford analysis, Riemannian geometry and the electromagnetic field, in: Essays on the Formal Aspects of Electromagnetic Theory, A. Lakhtakia (ed.), World Scientific Publ. Co., 1993, pp. 533-558.

[6] - and L. Wojtczak in cooperation with S. Koshi and O. Suzuki, Stochastic mechanics of particle systems in Cliford-analytical formulation related to Hurwitz pairs of bidimension (8, 5), in: Deformations of Mathematical Structures, J. Lawrynowicz (ed.), Kluwer Academic Publishers, Netherlands, 1994, 213-262.

[7] J. C. Le Bossé, J. J. Lopez, J. Rousseau, I. Zasada and L. Wojtczak, Statistical aspects of the diffuse LEED problem, J. Phys.: Condens. Matter, 2, (1990), 3143-3165.

[8] R. Lipowsky and W. Speth, Semi-infinite systems with first-order bulk transitions, Phys. Rev. B28 No. 7, (1983), 3983-3993.

[9] B. Pluis, D. Frenkel and J. F. van der Veen, Surface-induced melting and freezing II. A semiempirical Landau-type model, Surf. Sci., 239, (1990), 282-300.

[10] J. Rembieliński and K. Smoliński, Non-commutative quantum dynamics, Modern Physics Letters A, No. 35 8, (1993), 3335-3343.

[11] A. Urbaniak-Kucharczyk, Spin polarization transport in thin magnetic film, in: Deformations of Mathematical Structure, J. Ławrynowicz (ed.), Kluwer Academic Publ., Netherlands, 1994, 327-340.

[12] I. Zasada and L. Wojtczak, Topological correlations in the surface melting conditions, Bull. Soc. Sci. Lettres Łódź, Sér. Rech. Déform. 18, (1995), 93-108. 